\title{
Complications Following Prostate Biopsy: A Single Centre Five Year Review
}

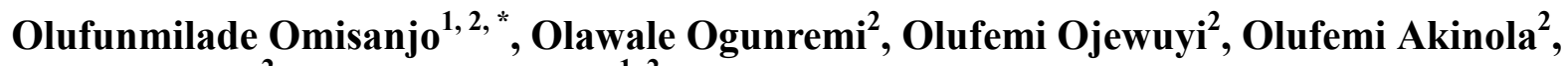 \\ Fatai Balogun ${ }^{2}$, Stephen Ikuerowo ${ }^{1,2}$ \\ ${ }^{1}$ Department of Surgery, Lagos State University College of Medicine, Ikeja, Nigeria \\ ${ }^{2}$ Department of Surgery, Lagos State University Teaching Hospital, Ikeja, Nigeria
}

Email address:

sanjofunmi@yahoo.com (O. Omisanjo)

${ }^{*}$ Corresponding author

\section{To cite this article:}

Olufunmilade Omisanjo, Olawale Ogunremi, Olufemi Ojewuyi, Olufemi Akinola, Fatai Balogun, Stephen Ikuerowo. Complications Following Prostate Biopsy: A Single Centre Five Year Review. International Journal of Clinical Oncology and Cancer Research. Vol. 4, No. 6, 2019, pp. 45-48. doi: 10.11648/j.ijcocr.20190406.11

Received: September 16, 2019; Accepted: November 13, 2019; Published: November 20, 2019

\begin{abstract}
Background: Though prostate biopsy is generally a safe procedure, it can be associated with complications. It is important to document the complication rates and identify potential risk factors for these complications. The aim of this study was therefore to investigate the complication rates following prostate biopsy at the Lagos State University Teaching Hospital Ikeja Lagos Nigeria over a 5 year period from January 2012 to December 2016. Patients and Methods: This was a retrospective study in which the clinical records of all the patients who had prostate biopsy at the Lagos State University Teaching Hospital Ikeja, Lagos Nigeria over a 5 year period between January 2012 and December 2016 were retrieved and analyzed. Results: The clinical records of a total of 258 patients were available for review. The mean age was 68.2 years (range 45 to 81 years). The mean and median PSA values were $560 \mathrm{ng} / \mathrm{ml}$ and $57 \mathrm{ng} / \mathrm{ml}$ respectively (range 2.05 to $15,400 \mathrm{ng} / \mathrm{ml}$ ). The prostate biopsy was transrectal and digitally guided in all cases. All the patients had empirical intravenous prophylactic antibiotics with intravenous ciprofloxacin $500 \mathrm{mg}$ stat and were discharged on oral ciprofloxacin $500 \mathrm{mg}$ bd and oral metronidazole $400 \mathrm{mg}$ tds for one week. All the patients had a caudal block. One hundred and seventeen $(45.3 \%)$ had a comorbidity. The mean prostate size was $109 \mathrm{gms}$ (range $16-146 \mathrm{gms}$ ). The size of the trucut needle used was size 16 in 121 patients $(46.9 \%)$ and size 18 in 125 patients (48.4\%). The mean number of biopsy cores taken was 10 (range 4 to 15 ). The histological diagnosis was carcinoma of the prostate in 154 patients $(59.7 \%)$ and benign prostatic hyperplasia in 100 patients $(38.8 \%)$. Twenty four patients $(9.3 \%)$ had complications. The complications were sepsis $(3.1 \%)$, rectal bleeding $(2.3 \%)$, haematuria $(2.3 \%)$ and acute urinary retention $(1.6 \%)$. Thirteen patients needed hospitalization $(5 \%)$. There was no mortality. The incidence of sepsis was statistically significantly higher with increasing the number of cores taken $(\mathrm{p}=0.000)$, but there was no significant difference in the incidence of sepsis with the size of the trucut needle used $(p=0.299)$ or the presence of morbidity $(p=0.503)$. Conclusion: Though the complication rates following prostate biopsy remain low, increasing number of prostate cores taken is a risk factor for adverse events. We therefore recommend reducing the number of prostate cores taken in patients with advanced prostate cancer with high tumour volume in order to further reduce the risk of prostate biopsy complications in our environment.
\end{abstract}

Keywords: Prostate Biopsy, Complications, Sepsis

\section{Introduction}

Prostate biopsy is one of the most common procedures performed by urologists. This is because prostate cancer remains a leading cancer diagnosis and cause of cancer related deaths amongst men worldwide. Prostate cancer is the most common malignancy in the adult Nigerian male with reports suggesting it accounts for $19.6 \%$ of all cancer cases in Nigeria [1, 2].

A prostate biopsy is needed to make a histological diagnosis whenever prostate cancer is suspected following an abnormal digital examination, elevated serum prostate specific antigen (PSA) or both. The prostate biopsy technique 
as well as the various indications and results have been widely reported $[3,4]$. Though a prostate biopsy is generally a simple and safe procedure with low mortality [4], it is not without possible complications. These complications can range from mild and self-limiting, to severe and potentially life-threatening. Since most prostate biopsies are performed transrectally, infectious complications such as fever, urinary tract infection, epididymoorchitis and sepsis may arise from the introduction of rectal bacteria into the prostate, the urinary tract or bloodstream [5, 6]. The reported noninfectious complications that may arise include haematuria in up to $14.5 \%$ of cases and rectal bleeding in up to $2.2 \%$ of cases [7]. Other reported complications include haemospermia, perineal pain and acute urinary retention. While some of these complications may persist for up to two weeks, there is usually a progressive decrease in their severity with time. [8]

It is important that these complications be borne in mind whenever patients are being prepared and counselled for prostate biopsy. There are few reports available in Nigeria on prostate biopsy complication rates, thus there are limited local data to guide urologists while taking consent for prostate biopsy. Some of the fears that patients have about prostate biopsy complications may actually be exaggerated. The aim of this study was therefore to investigate the complication rates following prostate biopsy at the Lagos State University Teaching Hospital Ikeja Lagos Nigeria over a 5 year period from January 2012 to December 2016.

\section{Patients and Methods}

This was a retrospective study in which the clinical records of all the patients who had prostate biopsy at the Lagos State University Teaching Hospital Ikeja, Lagos Nigeria over a 5 year period between January 2012 and December 2016 were retrieved. The data analysed were the age of the patient, serum PSA, prostate size, presence of comorbidity, cadre of the doctor performing the biopsy, antibiotic prophylaxis, type of anaesthesia, size of trucut needle used, number of prostate cores taken, histological diagnosis, presence of complication and mortality. The data were expressed as means and medians, and analysis was by Statistical Package for Social Sciences (SPSS) version 20.0 for windows. We evaluated if the patient's age, presence of comorbidity, trucut needle size and the number of prostate cores taken were risk factors for complications following prostate biopsy. The test for statistical significance was carried out using the Fischer's exact and Chi square test, with a $P$ value $<0.05$ considered significant.

\section{Results}

The clinical records of a total of 258 patients were available for review. The age distribution of the patients is as shown in Table 1 with a mean age of 68.2years (range 45 to 81 years).
Table 1. Patients' Age Distribution.

\begin{tabular}{lll}
\hline Age (years) & Frequency & Percentage \\
\hline$\leq 50$ & 2 & 0.8 \\
$51-60$ & 31 & 12.0 \\
$61-70$ & 115 & 44.6 \\
$71-80$ & 89 & 34.5 \\
$81-0$ & 21 & 8.1 \\
Total & 258 & 100 \\
\hline
\end{tabular}

The mean and median PSA values were $560 \mathrm{ng} / \mathrm{ml}$ and $57 \mathrm{ng} / \mathrm{ml}$ respectively (range 2.05 to $15,400 \mathrm{ng} / \mathrm{ml}$ ). The prostate biopsy was transrectal and digitally guided in all cases. All the patients had empirical intravenous prophylactic antibiotics with intravenous ciprofloxacin 500mg stat and were discharged on oral ciprofloxacin $500 \mathrm{mg}$ bd and oral metronidazole $400 \mathrm{mg}$ tds for one week. All the patients had a caudal block. One hundred and seventeen (45.3\%) had a comorbidity. The commonest comorbidities were hypertension ( $\mathrm{n}=86,33.3 \%)$, diabetes $(\mathrm{n}=9,3.4 \%)$, and patients with both hypertension and diabetes $(n=22,8.5 \%)$. The mean prostate size was $109 \mathrm{gms}$ (range $16-146 \mathrm{gms}$ ). Most of the prostate biopsies were done by resident doctors $(\mathrm{n}=204,79.1 \%)$ while $20.9 \%(\mathrm{n}=54)$ were carried out by consultants. The size of the trucut needle used was size 16 in 121 patients $(46.9 \%)$ and size 18 in 125 patients $(48.4 \%)$. The needle size was not stated in 12 patients $(4.7 \%)$. (Figure 1)

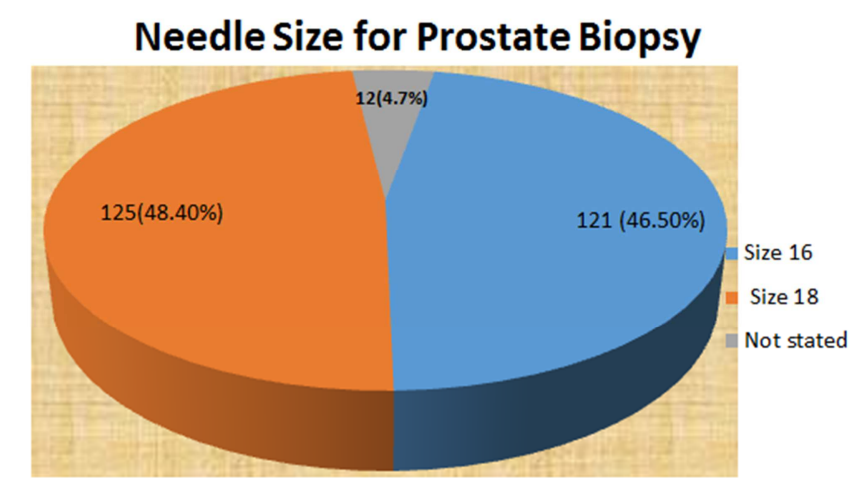

Figure 1. Needle Size for Prostate Biopsy.

Most of the patients $(n=196,76 \%)$ had between 8 and 12 prostatic cores taken and the mean number of biopsy cores taken was 10 (range 4 to 15). (Figure 2)

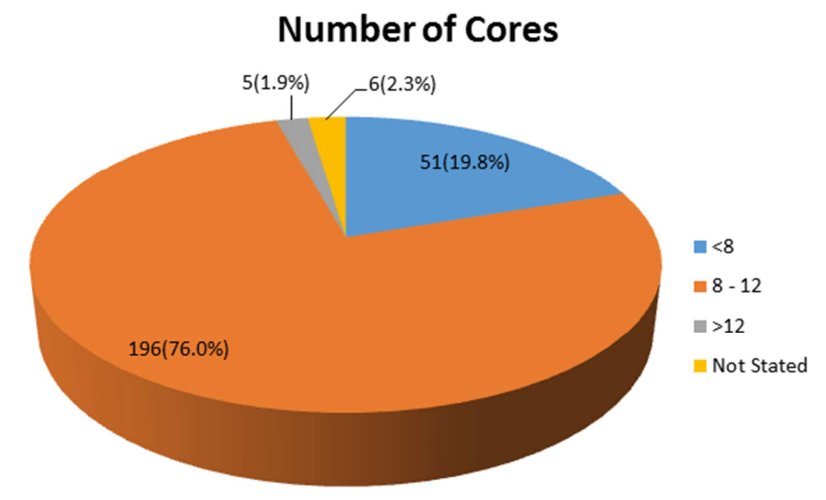

Figure 2. Number of Cores. 
The histological diagnosis was carcinoma of the prostate in 154 patients $(59.7 \%)$ and benign prostatic hyperplasia in 100 patients $(38.8 \%)$. (Table 2$)$

Table 2. Histological Diagnosis.

\begin{tabular}{lll}
\hline Diagnosis & Frequency & Percentage \\
\hline Carcinoma of the Prostate & 154 & 59.7 \\
Benign Prostatic Hyperplasia & 100 & 38.8 \\
Prostatic Intraepithelial Neoplasia & 2 & 0.8 \\
No Histology/Inadequate Specimen & 2 & 0.8 \\
Total & 258 & 100 \\
\hline
\end{tabular}

Twenty four patients (9.3\%) had complications. The complications were sepsis $(3.1 \%)$, rectal bleeding $(2.3 \%)$, haematuria $(2.3 \%)$ and acute urinary retention $(1.6 \%)$. Thirteen patients needed hospitalization $(5 \%)$. There was no mortality. (Table 3)

Table 3. Complications following Prostate Biopsy.

\begin{tabular}{lll}
\hline Complication & Frequency & Percentage \\
\hline No Complication & 234 & 90.7 \\
Rectal Bleeding & 6 & 2.3 \\
Haematuria & 6 & 2.3 \\
Urinary Retention & 4 & 1.6 \\
UTI/Sepsis & 8 & 3.1 \\
Total & 258 & 100 \\
\hline
\end{tabular}

The incidence of sepsis was statistically significantly higher with increasing the number of cores taken $(p=0.000)$, but there was no significant difference in the incidence of sepsis with the size of the trucut needle used $(p=0.299)$ or the presence of morbidity $(\mathrm{p}=0.503)$. The incidence of rectal bleed correlated negatively with the patients' age $(p=0.014)$.

\section{Discussion}

Most of the patients in our study presented with high PSA values indicative of advanced disease in keeping with the findings of Badmus et al [9]. Though an ultrasound guided prostate biopsy is the standard method of carrying out a transrectal prostate biopsy [3, 4], lack of ready access to ultrasound in the operating room, clinics and wards means most of the prostate biopsies done in our setting are essentially digitally guided as shown by previous workers [10-12]. The fact that most of our patients also present with advanced disease [9] means the absence of ultrasound guidance may not be a major drawback in this group of patients with advanced disease since the patients already have extensive prostatic involvement by the tumour. Our pick up rate for carcinoma of the prostate of $59.7 \% \%$ was higher than the $46 \%$ reported by Shittu et al [11].

We gave a caudal block as the means of anaesthesia and this was satisfactory for pain control in the majority of the patients. Previous studies have shown caudal block to be an effective means of anaesthesia for minor perineal procedures [13-15].

Our use of ciprofloxacin as prophylaxis is in line with the global recommendation and practice involving the use of a quinolone as the agent of first choice in the prevention of infective complications of prostate biopsy [16].
Our reported incidence of rectal bleeding of $2.3 \%$ was similar to the $2.2 \%$ reported by Ecke et al but lower than the $5.2 \%$ reported by Shittu et al [7, 11]. The incidence of haematuria of $2.3 \%$ we found was also less than the reported $5.2 \%$ by Shittu et al and $14.5 \%$. by Ecke et al $[7,11]$. We averagely used a size $16 \mathrm{G}$ or $18 \mathrm{G}$ needle for prostate biopsy which is smaller than the 14G reported by Shittu et al [11]. This may have contributed to the less risk of bleeding we found. We however did not find any statistically significant difference in the complication rates between the use of size $16 \mathrm{G}$ needle and the use of size $18 \mathrm{G}$ needle. A previous work by Cicione et al also did not show the use of size 16 needle to increase prostate biopsy morbidity over the use of size 18 [17].

The incidence of sepsis (of 3.1\%) and hospitalization rate (of 5\%) we recorded were higher than the respective rates of $0.1-0.5 \%$ and $1.6 \%$ reported by Chiang et al [18], but our reported incidence of sepsis was similar to the $4.3 \%$ reported by Shittu et al [11]. Some conditions have been documented as being risk factors for sepsis and these include mainly urethral catheterization and diabetes mellitus as reported by Lange et al and Simsir et al [19, 20]. We however did not find any significant difference in the complication rates (for bleeding or for sepsis) between the patients who had comorbidities (hypertension, diabetes mellitus) and those without comorbidities $(\mathrm{P}=0.503)$. Though it has been reported that the rates of discomfort and major complications do not depend on the number and site of punctures made with the biopsy needle and that their incidence is not higher in patients submitted to an initial biopsy or rebiopsy after some weeks [21, 22], we did find that the risk of sepsis was significantly higher with increasing the number of prostate cores taken. The plausible reasons for this difference in findings may be due to the more advanced disease in our patients with higher PSA, bigger prostate or the type of prophylactic antibiotic regimen used.

The prescribed number of prostate cores to be taken during a biopsy is generally recommended as 10 to 12 [23]. This helps to improve the chances of getting a positive core especially for patients with limited amount of tumour present. Also the percentage of positive cores may be important in disease stratification, prognostication and treatment planning in patients with early prostate cancer. It however does not appear that this argument or advantage of taking multiple cores holds for patients with advanced disease as is the case for most of the patients in our environment. Most of these patients with advanced disease already have a high tumour burden which can be detected histologically with few prostatic cores. Taking several prostatic cores in patients with advanced disease may not give any additional actionable information beyond the establishment of histological diagnosis.

In the light of our finding of a significantly increased risk of sepsis with increasing number of prostatic cores, we recommend that patients with advanced prostate cancer in our environment need not have multiple prostate cores taken for biopsy as the important information needed in this group of patients is the establishment of a histological diagnosis 
which can be achieved sometimes with even a single prostatic core. More studies will be needed to define what should be the ideal number of prostate cores to be taken for this group of patients in order to optimize histological yield on the one hand and minimize the risk of sepsis on the other. The negative correlation of rectal bleeding that we found with age will also require further investigation. Brewster et al had identified advancing age as a risk factor for adverse events following prostate biopsy. [24]

\section{Conclusion}

Though the complication rates following prostate biopsy remain low, the risk of sepsis is significantly higher with increasing number of prostatic cores in patients with advanced prostate cancer. Considering that most of the patients in our environment with carcinoma of the patient present late with high volume disease, we recommend a protocol of reducing the number of prostate cores taken in this group of patients with advanced prostate cancer with high tumour volume in order to further reduce the risk of prostate biopsy complications.

\section{Conflicts of Interest}

The authors declare no conflicts of interest.

\section{References}

[1] Parkin DM, Bray F, Ferlay J, Pisani P. Estimates of worldwide burden of cancer in 2002, Globocan 2002. CA Cancer J Clin. 2005; 55 (2): 74-108.

[2] Ogunbiyi JO, Shittu OB. Increased incidence of prostate cancer in Nigeria. Journal of National Medical Association 1999; 91 (3): 159-164.

[3] Scherr DS, Eastham J, Ohori M, et al. Prostate biopsy techniques and indications: when, where, and how? Semin Urol Oncol. 2002; 20: 18-31.

[4] Smeenge M, de la Rosette JJ, Wijkstra H. Current status of transrectal ultrasound techniques in prostate cancer. Curr Opin Urol. 2012; 22: 297-302.

[5] Zani EL, Clark OA, Rodrigues Netto N Jr. Antibiotic prophylaxis for transrectal prostate biopsy. Cochrane Database Syst Rev 2011; 5: CD006576.

[6] Liss MA, Chang A, Santos R, et al. Prevalence and significance of fluoroquinolone resistant Escherichia coli in patients undergoing transrectal ultrasound guided prostate needle biopsy. J Urol 2011; 185: 1283.

[7] Ecke TH, Gunia S, Bartel P, et al. Complications and risk factors of transrectal ultrasound guided needle biopsies of the prostate evaluated by questionnaire. Urol Oncol. 2008; 26: 474-478.

[8] Loeb S, Carter HB, Berndt SI, et al. Complications after prostate biopsy: data from SEER-Medicare. J Urol. 2011; 186: 1830-1834.

[9] Badmus TA, Adesunkanmi AR, Yusuf BM et al. Burden of prostate cancer in southwestern Nigeria. Urology 2010; 76 (2): 412-6.

[10] Udeh E. I, Amu O. C., Nnabugwu I. I., Ozoemena O. F. N. Transperineal versus tranrectal prostate biopsy: our findings in a tertiary health institution. Nigerian Journal of Clinical Practice. 2015: 18 (1); 110-114.

[11] Shittu O. B, Kamara T. B. Transrectal biopsy of the prostate gland in Ibadan. The Nigerian Journal of Surgical Research. 2001: 3 (4); 159-164.

[12] Eke N. Antibiotics prophylaxis for digital-guided transrectal tru-cut needle biopsy of the prostate. West Afr J Med. 2006; 25 (4): 262-265.

[13] Okeke LI. Day case transurethral prostatectomy in Nigeria. West Afr J Med. 2004 Apr-Jun; 23 (2): 128-30.

[14] Ikuerowo SO, Popoola AA, Olapade-Olaopa EO, Okeke LI, Shittu OB, Adebayo SA, Sanusi AA. Caudal block anesthesia for transrectal prostate biopsy. Int Urol Nephrol. 2010 Mar; 42 (1): 19-22. Epub 2007 Feb 22.

[15] Wang N, Fu Y, Ma H, Wang J, Gao Y. Advantages of caudal block over intrarectal local anesthesia plus periprostatic nerve block for transrectal ultrasound guided prostate biopsy. Pak J Med Sci. 2016 Jul-Aug; 32 (4): 978-82. doi: 10.12669/pjms.324.9823.

[16] Son KC, Chung HS, Jung S, Kim MS, Hwang EC, Kim JW, Kwon DD. Trial Comparing a Combined Regimen of Amikacin and Ciprofloxacin to Ciprofloxacin Alone as Transrectal Prostate Biopsy Prophylaxis in the Era of High Fluoroquinolone-Resistant Rectal Flora. J Korean Med Sci. 2018 Apr 9; 33 (15): e113.

[17] Cicione A, Cantiello F, De Nunzio C, Tubaro A, Damiano R. Prostate biopsy quality is independent of needle size: A randomized single-center prospective study. Urol Int 2012; 89: 57-60.

[18] Chiang IN, Chang SJ, Pu YS, et al. Major complications and associated risk factors of transrectal ultrasound guided prostate needle biopsy: a retrospective study of 1875 cases in Taiwan. J Formos Med Assoc. 2007; 106: 929-934.

[19] Lange D, Zappavigna C, Hamidizadeh R et al. Bacterial sepsis after prostate biopsy- a new perspective. Urology 2009; 74: 1200.

[20] Simsir A, Kismali E, Mammadov R, et al. Is it possible to predict sepsis, the most serious complication in prostate biopsy? Urol Int. 2010; 84: 395-399.

[21] Rodríguez LV, Terris MK. Risks and complications of transrectal ultrasound guided prostate needle biopsy: a prospective study and review of the literature. J Urol. 1998; 160: 2115-2120.

[22] Djavan B, Waldert M, Zlotta A, et al: Safety and morbidity of first and repeat transrectal ultrasound-guided prostate needle biopsies: Results of a prospective European prostate cancer detection study. J Urol 2001; 166: 856-860.

[23] Donovan, J., et al. Prostate Testing for Cancer and Treatment (Protec T) feasibility study. Health Technol Assess, 2003. 7: 1.

[24] Brewster DH, Fischbacher CM, Nolan J, Nowell S, Redpath D, Nabi G. Risk of hospitalization and death following prostate biopsy in Scotland. Public Health. 2017 Jan; 142: $102-110$. 